\title{
REFLEXÕES EM TEMPOS DE PANDEMIA: UM OLHAR SOBRE O TURISMO NO AMAZONAS
}

\author{
REFLECTIONS IN TIMES OF PANDEMIC: A LOOK AT TOURISM IN AMAZONAS
}

REFLEXIONES EN TIEMPOS DE PANDEMIA: UNA MIRADA AL TURISMO EN AMAZONAS

\section{RESUMO}

Este artigo busca caracterizar os impactos da pandemia do novo coronavírus (COVID-19) no setor do turismo em doze municípios no estado do Amazonas. Para isto, foi realizada pesquisa bibliográfica e documental com levantamento de dados públicos como finanças públicas, número de empresas e empregos do setor e informações sobre voos, relativos aos primeiros semestres de 2019 e 2020, comparativamente. A seleção dos municípios de interesse utilizou, a priori, o Mapa do Turismo Brasileiro (2019-2021) elaborado pelo Ministério do Turismo (MTur). Os principais resultados indicam que houve um aumento na receita pública do setor de turismo entre 2019 e 2020, ainda que nem todos os municípios tenham disponibilizado seus dados. Além disso, houve um esvaziamento nos aeroportos e diminuição de voos, queda no número de empregos, mesmo diante do aumento no número de empresas no setor. $O$ setor do turismo foi extremamente afetado pela pandemia, sendo que sua reativação será complexa, dada a precariedade e insuficiência dos municípios estudados com relação a suas estruturas sanitárias e ao oferecimento de formas seguras e rápidas no controle e tratamento da pandemia.

Palavras-chave: Turismo. COVID-19. Impactos. Amazonas.

\section{ABSTRACT}

The objective of this article is to characterize in a comparative manner the impacts of the pandemic of the new coronavirus (COVID-19) on the tourism sector in 12 municipalities in the State of Amazonas. For this purpose, has been done a bibliographic and documentary research with public data, such as public finances, number of companies and jobs in the tourism sector, flight information, for the first half of 2019 and the first half of 2020. The municipalities were selected based on the Brazilian Tourism Map (2019-2021) prepared by the Ministry of Tourism (MTur). The main results indicate that there was an increase in the revenue of the tourism sector between 2019 and 2020, however, not all municipalities have available data. Moreover, the airports were empty and there was a decrease in flights and in the number of jobs generated in this sector, although the number of tourist companies there was increase. It is concluded that the tourism sector was extremely affected by the pandemic, and this activity reactivation will be difficult considering the precariousness and insufficiency of municipalities' health infrastructure and lack of safe procedures and effective way to control and treat the pandemic.

Keywords: Tourism. COVID-19. Impacts. Amazon.

\section{RESUMEN}

Este artículo busca caracterizar, de manera comparativa, los impactos de la pandemia del nuevo coronavirus (COVID-19) en el sector turístico en 12 municipios del estado de Amazonas. Para ello, se desarrolla una investigación bibliográfica y documental que involucra a datos públicos oficiales del primero semestre de 2019 y el primero semestre de 2020 (finanzas públicas, número de empresas y empleos en el sector, informaciones sobre vuelos y aeropuertos). La selección de los municipios de interés se utiliza, a priori, el Mapa del Turismo Brasileño (2019-2021) elaborado por el Ministerio del Turismo (MTur). Los principales resultados indican que hubo un aumento en el ingreso público del sector del turismo en el periodo analizado, aún que los datos no están disponibles para todos los municipios. Además, se reconoce que, junto al vaciamiento en los aeropuertos, hubo caída en
Thais Zucheto de Menezes ${ }^{a}$

Susy Rodrigues Simonetti ${ }^{\text {b }}$

Arístocles Rannyeri

Nascimento de Lima ${ }^{\text {b }}$

D Deborah Rayanne Cabral Nogueira ${ }^{\mathrm{c}}$

a Universidade de São Paulo (USP), São Paulo, SP, Brasil

${ }^{\mathrm{b}}$ Universidade Estadual do Amazonas (UEA), Manaus, AM, Brasil

' Universidade Federal do Amazonas (UFAM), Manaus, AM, Brasil

DOI: $10.12957 /$ geouerj.2021.61315

Correpondência: zucheto@gmail.com

Recebido em: 20 mar. 2021

Aceito em: 29 jun.2021 
el número de empleos, a pesar del aumento en el número de empresas en el sector. Se sostiene que el sector turístico fue extremadamente afectado por la pandemia, siendo difícil su reactivación dada la precariedad e insuficiencia que presentan los municipios estudiados en sus estructuras sanitarias para brindar un marco de seguridad que actúe de forma rápida en el control y tratamiento de la pandemia.

Palabras-clave: Turismo. COVID-19. Impactos. Amazonas. 


\section{INTRODUÇÃO}

Em dezembro de 2019, a cidade de Wuhan, na China, foi atingida por uma doença causada pelo novo coronavírus (severe acute respiratory syndrome coronavirus -SARS-CoV-2), COVID-19 e, na sequência, 216 países, áreas ou territórios em todo o mundo foram afetados (WHO, 2019). No Brasil, a primeira infecção foi registrada pelo Ministério da Saúde em 26 de fevereiro de 2020 e, a primeira morte, em 17 de março (ZORZETTO, 2020), evoluindo rapidamente na segunda semana de junho do mesmo ano, quando o Brasil passou a ser o segundo país no mundo com o maior número de casos e de óbitos ocasionados pelo vírus (ALMEIDA et al, 2020). No Estado do Amazonas, a primeira confirmação de infecção pelo vírus foi identificada em 13 de março de 2020 (FVS/AM, 2021).

Desde o ano de 2019, o mapa do turismo mundial sofreu uma profunda transformação: a pandemia da COVID19 alterou o modo de vida global e modificou os fluxos turísticos drasticamente.

Em virtude da necessidade de se produzir estudos e, principalmente, conhecer os impactos da pandemia no setor do turismo, pesquisadores brasileiros e estrangeiros formaram um grupo de pesquisas intitulado "Turismo em Tempos de Pandemia: uma abordagem geográfica multi e trans-escalar". O grupo de pesquisas e o presente trabalho têm como base o conceito de impacto definido por Cruz (2020, p. 4):

\footnotetext{
poder-se-ia definir impacto como uma força emanada de uma ação ou um acontecimento capaz de trazer implicações diretas ou indiretas para o que ou para quem é atingido, sendo fundamental considerar que, quando socialmente sentidos, seus efeitos desdobram-se, dialeticamente, em ações, reações e contra-ações por parte da sociedade/grupo social/comunidade atingidos. É exatamente o que tem se passado no setor turismo após a deflagração da pandemia.
}

A atividade turística e a propagação do vírus da COVID-19 possuem uma característica em comum: a circulação. Neste sentido, compreendemos a importância da circulação no mundo contemporâneo e, assim como sinaliza Blanco (2015), é uma condição para o desenvolvimento do sistema de produção e fomenta uma demanda infinita e veloz dos agentes hegemônicos. Além disso, a circulação se desenvolve por meio do conjunto de relações sociais. Os territórios e suas atividades possuem dinâmicas próprias e em constante transformação, incluindo o turismo que foi e continua impactado pela circulação do vírus.

Ao longo das últimas décadas, as políticas governamentais de turismo no Amazonas foram direcionadas para a capital, Manaus, concentrando grande parte dos investimentos estaduais e federais. Dessa forma, em grande parte dos municípios do interior do Amazonas, o turismo mostra-se bastante incipiente e há um esforço, a cada ano, para que a maioria mantenha-se figurando no Mapa do Turismo Brasileiro.

Este artigo objetiva caracterizar os impactos da pandemia do novo coronavírus (COVID-19) no setor do turismo em doze municípios no estado do Amazonas. Para isto, foi realizado um recorte territorial baseado no Mapa do Turismo Brasileiro (2019-2021), elaborado pelo Ministério do Turismo (MTur). O Mapa divide o estado em sete Polos Regionais, totalizando 24 municípios. Para o presente estudo foram selecionados doze municípios: Barcelos (Polo Alto Rio Negro), 
Humaitá (Polo Madeira), Parintins (Polo Sateré), Itacoatiara (Polo Uatumã), Tabatinga (Polo Alto Solimões), Tefé (Polo Médio Solimões). Iranduba, Manacapuru, Manaus, Novo Airão, Presidente Figueiredo, Rio Preto da Eva (Polo Amazônico) também compõem da Região Metropolitana de Manaus (RMM).

Com base em uma pesquisa bibliográfica e documental, e por meio de formulário online, levantaram-se informações sobre finanças públicas, número de empresas do setor privado, empregos do setor turístico e fluxo de transporte aéreo. Todos os dados estão relacionados ao primeiro semestre dos anos de 2019 e 2020.

Para o levantamento dos dados primários junto aos órgãos municipais de turismo, utilizou-se um formulário, via Google Forms, composto por seis perguntas abertas acerca das estratégias da gestão municipal para enfrentamento dos impactos da COVID-19. Realizou-se contato com representantes das doze prefeituras municipais via telefone e e-mail, com membros de associações vinculadas ao turismo, empresários do setor e representantes dos órgãos oficiais de turismo com o intuito de obter informações ainda não publicadas sobre os impactos da pandemia em seus respectivos contextos. Este artigo está organizado da seguinte forma: são apresentados, de forma breve, os impactos da pandemia no mundo e no Brasil. Na sequência, são discutidos os impactos no estado do Amazonas, por meio da apresentação dos dados sobre finanças públicas, número de empresas turísticas do setor privado, empregos e fluxo de transporte aéreo, referentes ao primeiro semestre dos anos de 2019 e 2020. Por fim, evidenciam-se as diferenças do setor do turismo entre os municípios estudados e fazem-se recomendações.

\section{IMPACTOS DA PANDEMIA NO MUNDO E NO BRASIL}

Dentre as diversas crises regionais e globais que influenciaram os fluxos turísticos, com destaque para as econômicas, políticas, humanitárias, sanitárias, sem contar os furacões, terremotos, tsunamis, surtos de doenças e outras (PANOSSO NETTO et al, 2020), poucas trouxeram tantas incertezas quanto a atual pandemia que o mundo enfrenta.

Com o intuito de minimizar a pandemia, os países recorreram a medidas, como o isolamento domiciliar; quarentena voluntária e ou obrigatória; incentivo ao distanciamento social; fechamento de escolas, universidades, empresas, incluindo atividades não essenciais; cancelamento ou adiamento de eventos, desde debates políticos até os Jogos Olímpicos (GÖSSLING et al, 2020).

No caso do turismo, pandemias, como a causada pela COVID-19, afetam diretamente a mobilidade humana e, consequentemente, as atividades que dependem do fluxo de pessoas para a sua sobrevivência (Yang et al., 2020). Atualmente, o saldo negativo da pandemia no setor turístico supera o das grandes crises financeiras, como a Grande Depressão (1929-1933). Isso ocorre por um conjunto de fatores que impactam diretamente a vida das pessoas, dentre eles estão: recessão econômica e priorização para compras apenas de serviços essenciais; medidas restritivas impostas à cadeia produtiva de turismo, implicando em proibições para a administração de grandes massas e elevação de preços; 
aumento do medo do vírus e de aglomerações; desestabilização e falências no mercado de viagens - principalmente de empresas menores, bem como a redução de postos de trabalho; entre outros (BABA et al., 2020).

A situação que a sociedade está vivendo é sem precedentes e, para GRÖSSLING et al (2020),

\begin{abstract}
há muitas evidências de que a COVID-19 será diferente e transformadora para o setor de turismo. Os governos apenas começam a compreender que, ao contrário de outros setores empresariais, a receita do turismo está perdida permanentemente porque a capacidade não vendida - por exemplo, em acomodação - não pode ser comercializada nos anos subsequentes, com implicações correspondentes para o emprego no setor. (GRÖSSLING et al, 2020, p. 2, tradução nossa) ${ }^{1}$
\end{abstract}

Brito-Henriques (2020) e Higgins-Desbiolles (2020) afirmam que há demasiadas evidências da insustentabilidade do modelo dominante do turismo, sendo necessário refundá-lo. "Um turismo norteado não pelo critério do lucro das empresas, mas antes pelo interesse e benefício das comunidades visitadas, pela qualidade do emprego, e pela reconciliação ecológica e o respeito das fronteiras planetárias têm de ser o caminho" (BRITO-HENRIQUES, 2020, p. 208).

Repensar o modelo de desenvolvimento do turismo será uma oportunidade no pós-pandemia. Assim como aponta Harvey (2020, p. 16), "grande parte do modelo mais avançado no consumismo capitalista contemporâneo é inoperável nas condições atuais". O consumismo que motiva a economia capitalista contemporânea tem efeito nas condições ambientais, e a pandemia da COVID-19 está diretamente relacionada com as questões ambientais. "O progresso da COVID-19 exibe todas as características de uma pandemia de classe, de gênero e de raça" (Harvey, 2020, p. 17) e é inegável que a pandemia da COVID-19 evidenciou todas as questões que envolvem a mobilidade. A globalização e o turismo reforçaram o comportamento do vírus, fazendo-o circular livremente, avançando fronteiras pelos mais diversos recantos do planeta.

As redes de transporte são fundamentais para a circulação e conectividade do sistema de turismo. Isso é evidenciado pela redução drástica nos voos e os impactos nas viagens em navios de cruzeiro quando a pandemia foi declarada. Por meio de uma avaliação rápida dos impactos da COVID-19, Gössling et al (2020) identificaram que, a partir de 31 de março de 2020, mais de 90\% da população mundial estavam em países com algum nível de restrição para viagens internacionais, sendo que muitos desses países também tinham algum grau de restrição à circulação interna, incluindo viagens aéreas limitadas e a recomendação para permanecer em casa.

A UNWTO (2020) indica que o turismo precisará de dois e meio a quatro anos para voltar ao mesmo patamar de 2019, o que significa que até 2024 as consequências geradas por um ano de pandemia ainda estarão presentes. Além disso, a organização prevê que deverá ocorrer uma lenta recuperação da atividade devido às restrições de viagem pela lenta contenção do vírus, ambiente econômico desfavorável, falta de resposta coordenada entre os países, baixa confiança do consumidor (UNWTO, 2020).

\footnotetext{
1 "However, there is much evidence that COVID-19 will be different and transformative for the tourism sector. Governments only begin to understand that, unlike other business sectors, tourism revenue is permanently lost because unsold capacity - for instance in accommodation - cannot be marketed in subsequent years, with corresponding implications for employment in the sector".
} 
No Brasil, os efeitos mais expressivos da pandemia têm sido observados nos setores que trabalham diretamente com atendimento ao público, como comércio e serviços (SEBRAE, 2020). Segundo os dados do Cadastro Geral de Empregados e Desempregados (Caged), o setor de alojamento e alimentação teve um saldo de geração de empregos de -211.722 vagas, nos meses de março e abril de 2020. Quando comparadas às diferentes taxas de crescimento líquido setoriais entre os meses de 2020 e 2019, esse cenário persiste até o segundo semestre de 2020 (IPEA, 2020). No contexto do turismo mundial, o mesmo setor apresenta dificuldades de recuperação, fator que afeta diretamente o emprego de mulheres, uma vez que o setor de alojamento e alimentação tem $54 \%$ de sua força de trabalho constituída por pessoas do sexo feminino (UNWTO, 2020).

Conforme o agravamento da pandemia, o governo brasileiro optou pelo fechamento de fronteiras aéreas, aquaviárias e terrestres evitando a entrada de estrangeiros, como destaca Panosso Netto et al:

Excepcional e temporária, a medida de restrição pela Portaria n. 255, de 22 de maio de 2020 aplica recomendações da Agência Nacional de Vigilância Sanitária (ANVISA), considerando a declaração de emergência oficial da Organização Mundial da Saúde (OMS, 30/01/2020); prevenção de riscos em situações de emergência como princípio da Política Nacional de Segurança Pública; e controle das medidas de saúde frente à pandemia. Trata-se de uma medida importante, porém tomada com certo atraso temporal. (PANOSSO NETTO et al, 2020, p. 27)

A Fundação Getúlio Vargas - FGV (FGV, 2020) previu o início das atividades econômicas a partir de maio de 2020, a retomada gradual do turismo doméstico a partir de outubro de 2020, as viagens de negócios e eventos a partir de fevereiro de 2021 e a volta do turismo internacional a partir de meados de 2021. Estas análises abrangeram, principalmente, os setores de hospitalidade, transportes, bares e restaurantes, agenciamento e operadores de viagens, aluguel de imóveis e atividades recreativas, culturais e desportivas.

Trigo (2020) afirma que o setor mais atingido foi o agenciamento e a organização de viagens, seguido de transportes rodoviário e aéreo e sugere que a as perdas econômicas do turismo, em comparação ao PIB de 2019, totalizaram R\$ 116,7 bilhões no biênio 2020/2021, uma queda de 21,5\% na produção total do período.

O mesmo autor aponta algumas medidas governamentais para dar suporte ao setor, como "auxílios públicos, especialmente para o setor aéreo; revisão de contratos de concessão; crédito facilitado às pequenas e médias empresas; redirecionamento dos recursos e esforços para promoção de destinos domésticos [...]". (Trigo, 2020, p. 9).

Sobre as perspectivas para a retomada do turismo, Allis e de Paula (2020) observam que existem duas vertentes. A primeira retrata a volta ao normal mediante protocolos de biossegurança (vacinas, protocolos de convivência social, testes etc.) novos ou aperfeiçoados, assumindo que a pandemia é um evento intenso, porém necessária e desejavelmente superável. A segunda corrente entende que o turismo necessita de uma revisão, entendendo a pandemia como uma oportunidade para aplicar alguns princípios condizentes com a Agenda 2030 e com as políticas de turismo (decrescimento, trabalho decente, sustentabilidade), verticalizando a análise das dimensões do turismo, tendo em vista que a atividade ultrapassa a visão simplória do emaranhado de serviços e da satisfação do turista. 
Dentre os estados da federação, o Amazonas foi um dos primeiros em que a pandemia se alastrou causando desemprego, mortes e colapso do sistema público de saúde em seu vasto território.

\section{IMPACTOS DA PANDEMIA NO TURISMO NO ESTADO DO AMAZONAS}

De acordo com Aleixo et al (2020), Manaus teve a confirmação do primeiro caso da COVID-19, no estado do Amazonas, no dia 13 de março de 2020, mas a transmissão local da doença ocorreu a partir do dia 28 do mesmo mês. "Rapidamente a difusão da doença e o aumento da incidência pelos municípios em todas as regiões do estado provocaram o colapso do sistema de saúde estadual" (ALEIXO et al, 2020, p. 337).

O epicentro da pandemia no estado foi Manaus que, além de ser capital do estado, possui características que orientam a rede urbana. Manaus possui 52,75\% (2.219.580 de habitantes de acordo com o IBGE, 2020) da população total do estado (4.207.714 de habitantes, IBGE, 2020) e apresenta um alto fluxo de pessoas (incluindo povos indígenas e populações tradicionais) e produtos, principalmente por meio da circulação fluvial, com grande importância no estado, além da circulação aérea e rodoviária. Este alto fluxo de circulação contribuiu para a expansão do vírus a partir de Manaus para outros municípios do estado, ligados à capital por meio das redes urbanas, com destaque para Parintins, Tefé e Tabatinga (Schor e Oliveira, 2011).

Aleixo et al (2020) destacam que aeroportos, rodovias e portos, infraestruturas ligadas à fluidez no território, foram responsáveis pela difusão da doença. Os aeroportos e portos nas cidades mencionadas anteriormente, possibilitaram a expansão da doença de forma combinada no estado, e são considerados representativos em uma difusão de formato hierárquico.

Nos municípios da Região Metropolitana como Manacapuru, Iranduba, Itacoatiara, Novo Airão e Presidente Figueiredo, ligados por rodovia, a difusão da COVID-19 foi por contágio, e o gradativo aumento no número de casos foi facilitado pela proximidade e conectividade com a capital Manaus, "[...] os deslocamentos diários e semanais da população e o baixo isolamento e distanciamento social da população, foram fatores que contribuíram para difusão" (Aleixo et al, 2020).

É possível afirmar diante desse cenário, de acordo com Aleixo et al (2020, p. 4), que "a transmissão sofre influência da fricção do espaço, ou seja, uma cidade irradia a transmissão para outras no seu entorno". Os autores apontam que tanto a difusão hierárquica quanto a difusão por contágio “ocorrem pelo principal modal de transporte do estado do Amazonas, o fluvial" (Aleixo et al, 2020, p. 8). 
As cidades médias ${ }^{2}$ no estado do Amazonas possuem grande importância para a formação da rede urbana e para o fluxo da população. Schor e Costa (2007, p. 8) indicam que, na Amazônia, "as cidades médias constituem-se em novos vetores de crescimento econômico e demográfico sem, no entanto, afetar a primazia da metrópole". Além disso,

o dinamismo econômico e a estruturação intra-urbana das cidades na região Norte não estão relacionados ao patamar demográfico alcançado por essas cidades nas últimas décadas, mas principalmente à capacidade que possuem de responder às demandas regionais, seja do ponto de vista do capital, seja do ponto de vista da força de trabalho, tornando-se importantes nós de articulação de redes técnicas e de fluxos, tanto no contexto municipal quanto no contexto da mesorregião da qual fazem parte (SCHOR e COSTA, 2007, p. 8).

A Figura 1 ilustra a disseminação dos casos da COVID-19 no Amazonas em 15 de maio de 2020, período inicial da pandemia. É possível identificar a importância da malha hidrográfica, além da localização de cidades médias na difusão do vírus, que como indicado por Schor e Costa (2007), tornam-se nós de articulação de redes técnicas e de fluxos. No estado do Amazonas, muitas das atividades relacionadas ao setor do turismo apresentam vínculo com as cidades médias como, por exemplo, os doze municípios estudados.

Figura 1. Disseminação dos casos de COVID-19 no estado do Amazonas - 15 de maio de 2020. Fonte: Aleixo e Silva Neto, 2020

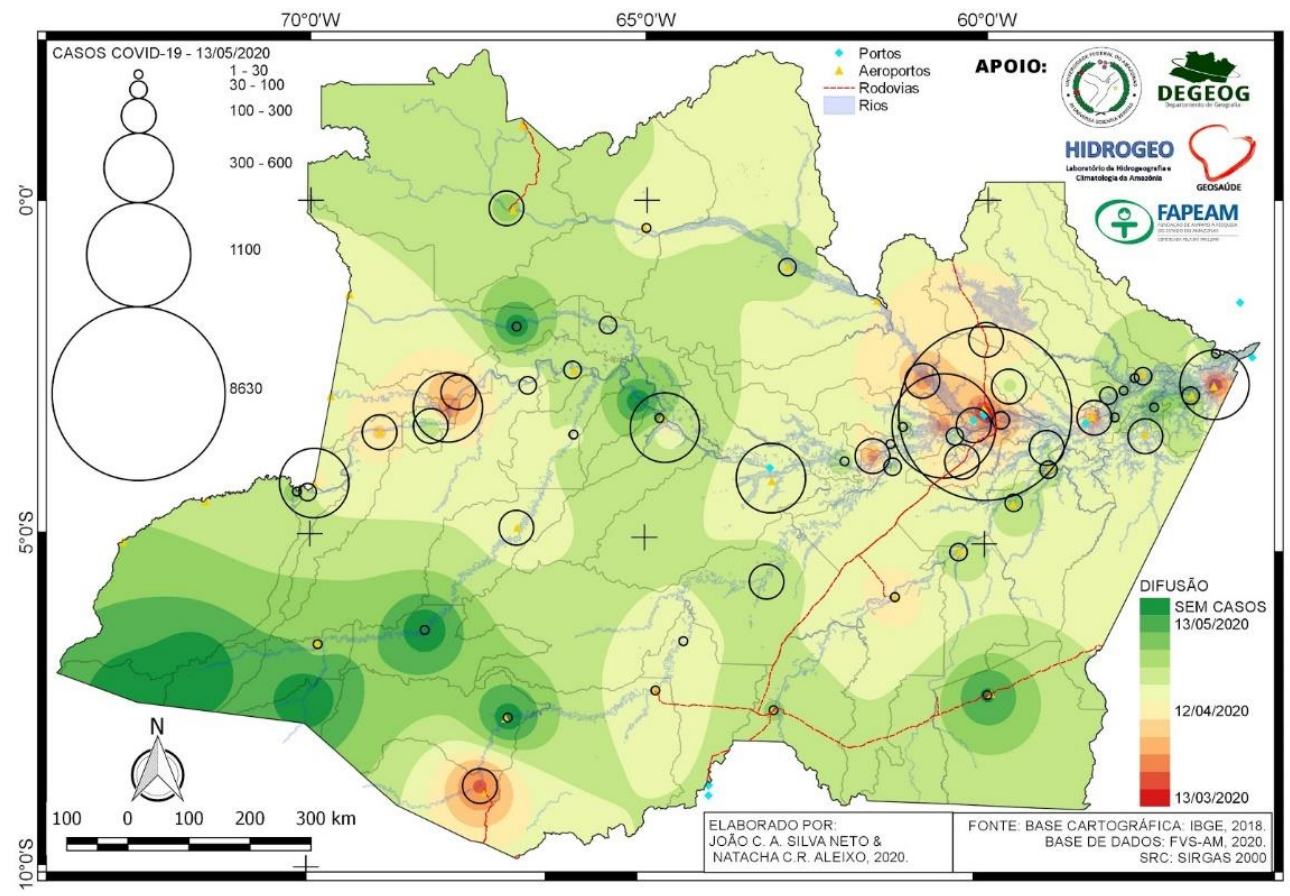

Aleixo et al (2020) salienta que a evolução progressiva dos casos no Amazonas ocorreu de forma distinta na capital e no interior. Manaus tem função de comando na rede urbana do estado e contribuiu para a expansão hierárquica da doença:

\footnotetext{
${ }^{2}$ Cidades médias são aquelas que possuem certa importância na estrutura urbano-regional, como o explicado por SPÓSITO, 2010, p. 53: "aquelas que desempenham papéis de intermediação no âmbito de sistemas urbanos simples ou complexos, o que as distingue das "cidades de porte médio", que são aquelas, assim classificadas, em função de um dado tamanho demográfico, que, num determinado país, é considerado como médio segundo esta ou aquela classificação ou tipologia.
} 
A função hierárquica é relacionada a posição da cidade na rede urbana e seu papel de centralidade, como por exemplo de uma metrópole que comenda a rede urbana. A hierarquia está aliada a oferta de serviços e produtos e na presença de infraestruturas como aeroportos, rodovias, portos que a conecta a outras cidades em várias escalas, desde a global até a local, possibilitando 'saltar escalas'. (ALEIXO et al, 2020, p. 4)

A pandemia impactou as economias municipais de formas distintas e o turismo não ficou alheio a este processo. Ações como o bloqueio da circulação da população e interrupção das atividades econômicas no estado tentaram colaborar para o não agravamento da pandemia pelo estado.

A Empresa Estadual de Turismo do Amazonas (Amazonastur) publicou, em 19 de junho de 2020, um conjunto de protocolos de biossegurança com medidas para a retomada do turismo, no entanto, nem os protocolos e nem o plano de reabertura do Amazonas apresentavam ações efetivas para garantir o controle e o tratamento da COVID-19 nos municípios do interior do estado (BARÉ et al, 2020).

Neste trabalho, realizado a partir de fontes bibliográficas e documentais, são apresentados dados referentes ao primeiro semestre dos anos de 2019 e 2020. Assim, foi possível a comparação dos cenários sem a presença da pandemia e com o início dela em 12 municípios do estado, sendo eles: Barcelos, Humaitá, Iranduba, Itacoatiara, Manacapuru, Manaus, Novo Airão, Parintins, Presidente Figueiredo, Rio Preto da Eva, Tabatinga e Tefé. A Figura 2 ilustra a os Polos Regionais Turísticos do estado e a localização dos municípios estudados.

Figura 2. Mapa dos Polos Regionais Turísticos do estado do Amazonas e localização dos municípios estudados. Fonte: IBGE (2021), MTur (2021). Elaborado por: Oliveira, Maria Rita Pelegrin. Software utilizado: ArcGis

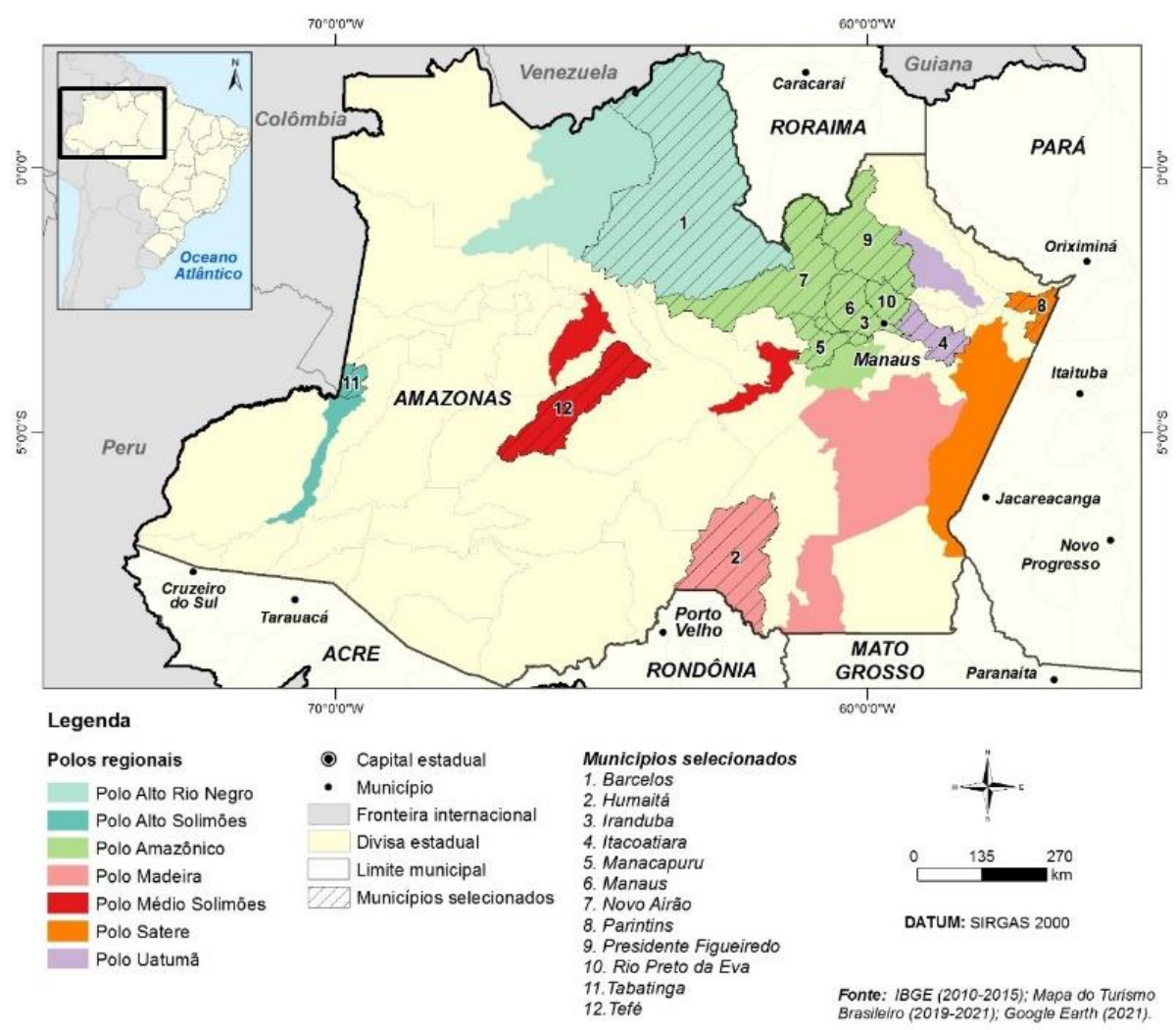


Mesmo com a indisponibilidade de alguns dados e considerando que as informações ainda se apresentam de forma incipiente, foi possível identificar impactos sobre finanças públicas, sobre o número de empresas turísticas do setor privado, geração de empregos e fluxo de transporte aéreo, conforme apresentado na sequência.

Finanças públicas

A análise das finanças públicas sobre o primeiro semestre de 2019 e de 2020 apresentou-se inconsistente com a ausência ou parcialidade de informações:

- Receitas gerais: não foram encontradas informações de dois municípios, Iranduba e Itacoatiara;

- Despesas gerais: apenas os municípios de Itacoatiara e Parintins não disponibilizaram as informações;

- Receita do setor turístico: foram disponibilizadas apenas por quatro municípios, Barcelos, Iranduba, Rio Preto da Eva e Tabatinga. Faltam dados para Humaitá, Manaus, Manacapuru, Novo Airão, Parintins, Presidente Figueiredo e Tefé;

- Despesa do setor turístico: foram disponibilizados dados apenas por oito municípios: Barcelos, Iranduba, Manaus, Manacapuru, Novo Airão, Parintins, Presidente Figueiredo e Rio Preto da Eva. Faltam dados para Humaitá, Itacoatiara, Tabatinga e Tefé.

Os dados obtidos estão apresentados nas Figuras 3 e 4, porém a indisponibilidade de alguns deles tornou a análise deficiente. A Figura 4 ilustra apenas dados dos municípios que disponibilizaram todos os valores de receitas e despesas para o setor do turismo nos primeiros semestres de 2019 e 2020 (Rio Preto da Eva, Iranduba e Barcelos).

Figura 3. Variação das receitas e despesas gerais (em mil R\$) - 1ㅇ semestre de 2019 e 2020. Fonte: https://transparenciamunicipalaam.org.br/. Elaborado por: Menezes, Simonetti, Nogueira, Lima

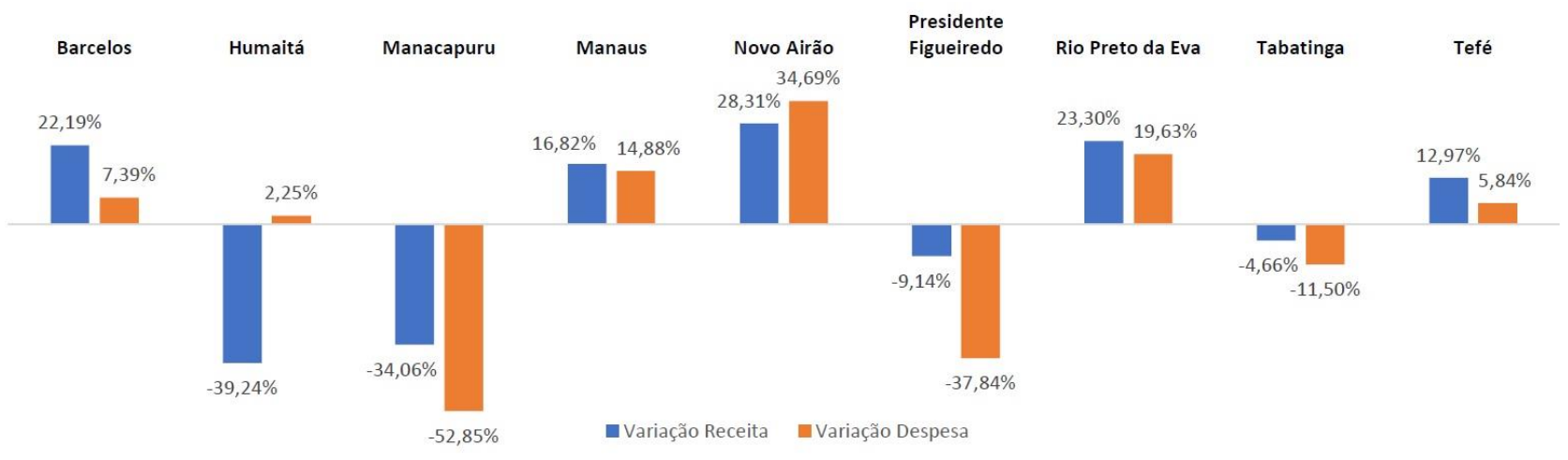


Figura 4. Receitas e despesas do setor de turismo (em mil R\$) - 10 semestre de 2019 e 2020. Fonte: https://transparenciamunicipalaam.org.br/. Elaborado por: Menezes, Simonetti, Nogueira, Lima

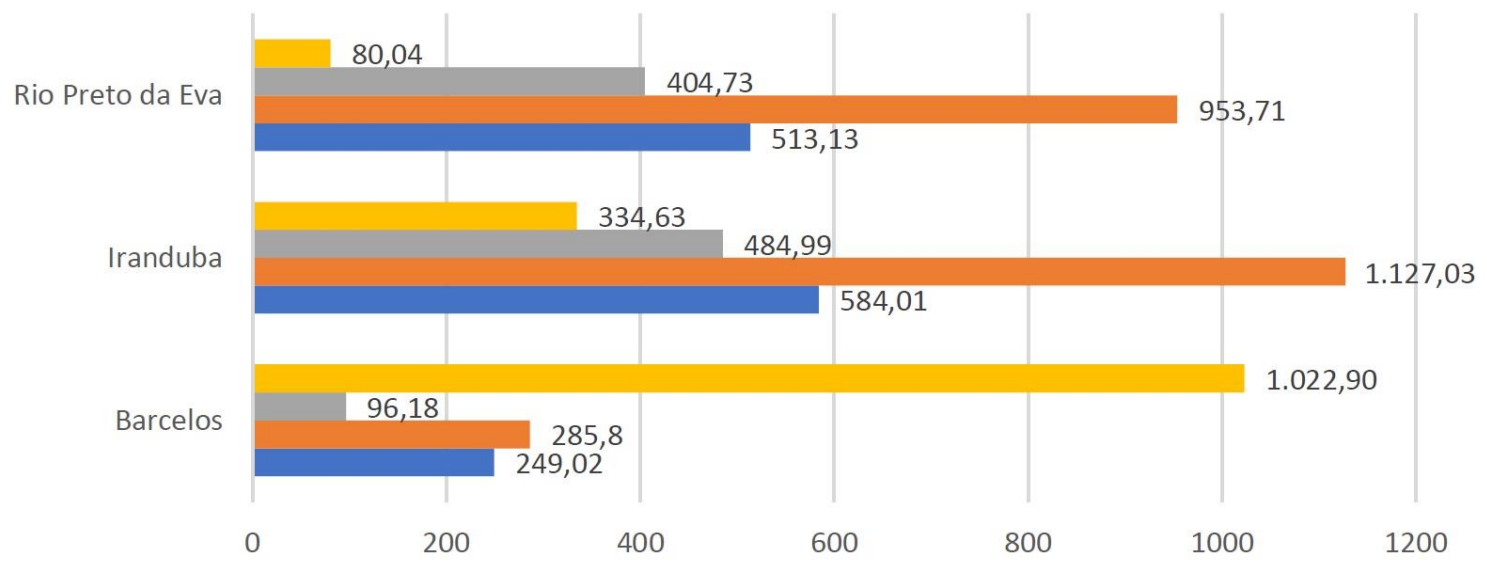

Despesa Setor Turismo 10 semestre 2020 - Despesa Setor Turismo 10 semestre 2019

— Receita Setor Turismo 1ㅇs semestre 2020 neceita Setor Turismo 1오 semestre 2019

Comparando os primeiros semestres de 2019 e 2020, os municípios que tiveram maior aumento na receita geral foram Novo Airão $(28,3 \%)$ e Rio Preto da Eva $(23,3 \%)$ e maior queda foram Humaitá $(39,2 \%)$ e Manacapuru (34,1\%). Com relação às despesas gerais, os municípios com maiores aumentos foram Novo Airão (34,7\%) e Rio Preto da Eva (19,6\%) e, com maior diminuição de despesas, foram Manacapuru (52,8\%) e Presidente Figueiredo (37,8\%), conforme indicado na Figura 3.

Sobre as finanças públicas ligadas ao setor do turismo (Figura 4), Iranduba e Rio Preto da Eva apresentaram um aumento de receita no setor de $93 \%$ e $86,9 \%$ respectivamente. E com relação às despesas do setor do turismo, os municípios de Barcelos apresentou aumento de 963,5\%. Rio Preto da Eva diminuiu suas despesas do setor do turismo em $80,2 \%$. Aqui destacamos o colossal aumento de despesas no município de Barcelos e a grande diminuição de despesas no município de Rio Preto da Eva.

No município de Barcelos, o site da transparência municipal indica que, no primeiro semestre de 2019, todas as despesas informadas foram relativas exclusivamente à manutenção da Secretaria de Turismo municipal, enquanto, no primeiro semestre de 2020, foram registradas despesas relacionadas à Manutenção de Projeto de Ecoturismo, Manutenção e Funcionamento do Fundo de Desenvolvimento Sustentável do Turismo de Pesca Esportiva. O registro destas despesas com manutenção de projetos em 2020 seria o motivo provável do elevado aumento nas despesas do setor do turismo.

A Lei da Transparência (Lei Complementar no 131, de 27 de maio de 2009) estabeleceu que deve ocorrer a disponibilização, em tempo real, de informações pormenorizadas sobre a execução orçamentária e financeira da União, dos Estados, do Distrito Federal e dos Municípios. Porém, durante a realização da pesquisa, não foi possível consultar o detalhamento dos valores apresentados, apenas os valores totais 
estavam disponíveis. $\mathrm{E}$, como destacado anteriormente, alguns municípios não disponibilizaram todos os valores de receitas e despesas. Além disso, não é possível determinar as causas do aumento ou redução das despesas, devido à indisponibilidade de informações sobre a tipologia dos valores declarados pelos municípios. Para compreensão dos valores apresentados e das grandes diferenças entre receitas e despesas, novas pesquisas devem acontecer junto aos órgãos municipais responsáveis.

\section{Empresas turísticas do setor privado e empregos}

O Quadro 1 indica o número de empresas turísticas do setor privado (setores de transporte, hotelaria, alimentação e no mercado de viagens e entretenimento) para o semestre dos anos de 2019 e 2020, segundo dados do DataSebrae. Os municípios de Manaus, Novo Airão e Rio Preto da Eva apresentaram redução no número de empresas, e os demais registraram aumento, com destaque para o município de Presidente Figueiredo, com registro de 106 novas empresas, aumento de 36,9\% entre o primeiro semestre de 2019 e de 2020, totalizando 393 no primeiro semestre de 2020.

Quadro 1. Empresas turísticas do setor privado para o 1o semestre dos anos de 2019 e 2020. Fonte: DataSebrae, 2019 e 2020

\begin{tabular}{|l|l|l|}
\hline Município & 10 semestre 2019 & 10 semestre 2020 \\
\hline Barcelos & 84 & 99 \\
\hline Humaitá & 187 & 220 \\
\hline Iranduba & 181 & 209 \\
\hline Itacoatiara & 271 & 283 \\
\hline Manacapuru & 221 & 239 \\
\hline Manaus & 11.483 & 11.253 \\
\hline Novo Airão & 143 & 127 \\
\hline Parintins & 314 & 351 \\
\hline Presidente Figueiredo & 287 & 393 \\
\hline Rio Preto da Eva & 158 & 126 \\
\hline Tabatinga & 181 & 198 \\
\hline Tefé & 116 & 190 \\
\hline
\end{tabular}

Uma hipótese para o aumento no número de empresas turísticas nos municípios citados é que aquelas, as quais operavam sem cadastro ou estavam ilegais junto aos órgãos oficiais, realizaram seu registro durante a pandemia do coronavírus devido à necessidade de apoio e dos incentivos do governo federal por parte dos empresários.

Um outro setor duramente afetado pelos impactos da pandemia foi o de empregos. De acordo com Secretaria de Estado de Desenvolvimento Econômico, Ciência, Tecnologia e Inovação do Estado do Amazonas (SEDECTI), entre os anos 2012 a 2014, houve aumento no número de empregos no estado, a queda de empregos no setor durou até 2017, sendo observada uma retomada no crescimento em 2018 (Figura 5). 0 ano de 2014 se destaca, pois ocorreu a Copa do Mundo de Futebol no Brasil, sendo Manaus uma das sedes. 
Figura 5. Evolução do emprego no turismo - Manaus e Brasil - 2012 a 2018. Fonte: SEDECTI, 2018. Elaborado por: Menezes, Simonetti, Nogueira, Lima

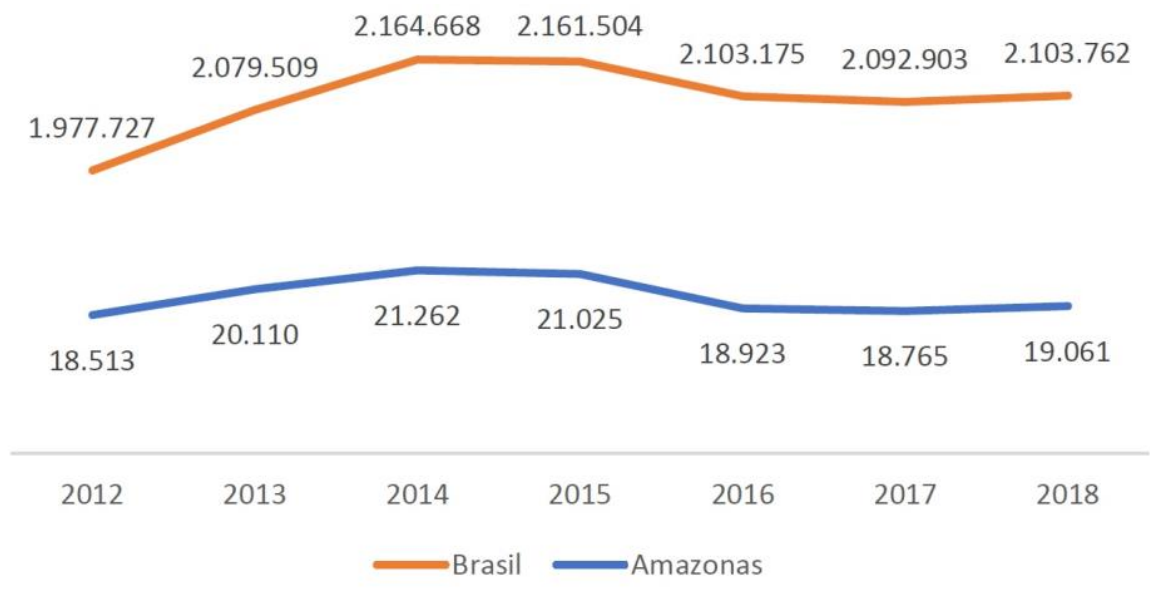

No entanto, com o início da pandemia em 2020, o setor de empregos ganhou uma nova feição. 0 Quadro 2 apresenta os dados sobre emprego formal nas $\mathrm{ACT}^{3}$ dos municípios estudados, sendo relevante para este trabalho a admissão e o desligamento de profissionais entre o primeiro semestre de 2019 e o primeiro semestre de 2020.

Quadro 2. Total de empregos nas Atividades Características do Turismo (ACTs). Fonte: Caged, 2020

\begin{tabular}{|l|l|l|l|l|}
\hline \multirow{2}{*}{ Município } & Admissão & \multicolumn{2}{l|}{ Desligamento } \\
\cline { 2 - 6 } & 1o Sem 2019 & 10 Sem 2020 & 10 Sem 2019 & 10 Sem 2020 \\
\hline Barcelos & 0 & 0 & 1 & 0 \\
\hline Humaitá & 19 & 16 & 16 & 25 \\
\hline Iranduba & 2 & 9 & 2 & 16 \\
\hline Itacoatiara & 31 & 16 & 34 & 24 \\
\hline Manacapuru & 13 & 4 & 13 & 9 \\
\hline Manaus & 2.628 & 1.938 & 2.661 & 3.442 \\
\hline Novo Airão & 12 & 6 & 9 & 15 \\
\hline Parintins & 9 & 2 & 9 & 8 \\
\hline Presidente Figueiredo & 3 & 2 & 7 & 2 \\
\hline Rio Preto da Eva & 2 & 3 & 14 & 6 \\
\hline Tabatinga & 13 & 8 & 10 & 10 \\
\hline Tefé & 5 & 4 & 6 & 18 \\
\hline
\end{tabular}

Observa-se que nos municípios de Iranduba e Rio Preto da Eva ocorreram mais admissões no primeiro semestre de 2020, comparativamente com o mesmo período de 2019 , sendo sete contratações a mais em Iranduba e uma contratação a mais em Rio Preto da Eva. Nos demais municípios, observa-se que, em 2020, houve menos admissões, sendo mais significativo em Parintins e Manacapuru, com 77,8\% e 69,2\% menos admissões no primeiro semestre de 2020, em comparação ao primeiro semestre de 2019. É importante

\footnotetext{
${ }^{3}$ As Atividades Características de Turismo (ACT) utilizadas no presente estudo foram agrupadas por principais Categorias/Subclasse de acordo com a seleção realizada pelo MTur, em articulação com o IBGE e IPEA, com base nos padrões recomendados pela OMT, na publicação "Recomendações Internacionais de Estatísticas de Turismo - RIET 2008 ".
} 
registrar ainda que, devido à pandemia, o Festival Folclórico de Parintins, evento de expressão nacional que atrai grande público, assim como eventos tradicionais de outros municípios do estado, não ocorreram em 2020, afetando diretamente a geração de emprego e renda nesses municípios.

Com relação às demissões, cinco municípios (Humaitá, Iranduba, Manaus, Novo Airão e Tefé) apresentaram números maiores no primeiro semestre de 2020, sendo que o número de demissões em Iranduba e Novo Airão foram mais significativos, com aumento de $700 \%$ e $66,7 \%$, respectivamente. Os demais municípios analisados apresentaram números menores, sendo que Presidente Figueiredo e Rio Preto da Eva apresentaram proporcionalmente menos demissões no período analisado, com $71,4 \%$ e $57,1 \%$ a menos, respectivamente.

\section{Fluxo de transporte aéreo}

Inicialmente, para a realização da análise dos impactos da pandemia no fluxo de transporte, pretendiase levantar dados referentes a três modais: fluvial, rodoviário e aéreo. Porém, a indisponibilidade de dados referentes aos transportes fluvial e rodoviário limitou a pesquisa e a avaliação dos dados sobre o transporte aéreo.

De acordo com Aleixo et al (2020), os aeroportos são infraestruturas ligadas à fluidez do território e contribuem para o processo de difusão da doença. Para Oliveira e Castilho $(2020$, p. 3), o transporte aéreo “pode ser visto como um vetor potencializador de alcance mundial [...]. Na perspectiva geográfica, é possível dizer que a pandemia revelou ainda mais disparidades no que tange às redes, principalmente a rede de transportes, vetor de mobilidade e de fluxos."

Sendo assim, avaliar os fluxos de transporte aéreo no estado do Amazonas permite compreender a difusão da doença por este modal. Além disso, possibilita compreender como a pandemia impactou na circulação do estado e em todas as atividades relacionadas direta ou indiretamente com o modal do transporte aéreo.

Entre os doze municípios estudados, apenas quatro possuem aeroportos: Manaus, Parintins, Tabatinga e Tefé, sendo que apenas Manaus possui fluxo de voos internacionais e é o portão de entrada da demanda turística do estado. O Quadro 3 apresenta o fluxo de transporte aéreo para os primeiros semestres de 2019 e 2020, com a indicação do número total de voos (chegadas e partidas) e o número total de embarques e desembarques. 


\begin{tabular}{|l|c|c|c|c|c|c|c|c|}
\hline \multirow{2}{*}{ Municípios } & \multicolumn{4}{|c|}{ N. de voos } & \multicolumn{3}{c|}{ N. desembarques/embarques } \\
\cline { 2 - 9 } & Cheg. & Part. & Cheg. & Part. & Desemb. & Emb. & Desemb. & Emb. \\
\cline { 2 - 9 } & \multicolumn{2}{|c|}{2019} & \multicolumn{2}{|c|}{2020} & \multicolumn{2}{|c|}{2019} & \multicolumn{2}{c|}{2020} \\
\hline $\begin{array}{l}\text { Manaus (Voos } \\
\text { Nacionais) }\end{array}$ & 6.024 & 6.190 & 3.977 & 4.002 & 659.941 & 671.506 & 383.826 & 386.367 \\
\hline $\begin{array}{l}\text { Manaus (Voos } \\
\text { Internacionais) }\end{array}$ & 626 & 645 & 429 & 451 & 31.522 & 31.460 & 14.015 & 12.644 \\
\hline Parintins & 401 & 400 & 155 & 155 & 10.456 & 10.305 & 1.234 & 3.783 \\
\hline Tabatinga & 179 & 178 & 87 & 87 & 16.200 & 14.453 & 8.148 & 6.887 \\
\hline Tefé & 194 & 192 & 124 & 123 & 8.012 & 7.409 & 4.915 & 3.783 \\
\hline
\end{tabular}

Houve queda significativa no número de voos (chegadas e partidas) e de embarques e de desembarques nos aeroportos de todos os municípios analisados entre o primeiro semestre de 2019 e 2020 . Os municípios que sofreram maior redução de voos (chegadas e partidas) foram Parintins, com queda de mais de $60 \%$; Tabatinga, com queda de mais de 55\%; e Tefé, com queda superior a $36 \%$.

Ao avaliar o número de desembarques e embarques, os municípios mais impactados foram Parintins, Manaus (voos internacionais) e Tabatinga. Parintins teve queda de $88 \%$ no desembarque e de $63 \%$ nos embarques; Manaus teve $56 \%$ de queda nos desembarques e $60 \%$ nos embarques, ambos no caso internacional; e Tabatinga teve queda de $50 \%$. nos desembarques e $52 \%$ nos embarques.

Este fenômeno está relacionado às medidas de diminuição de voos nacionais e, em alguns casos, à suspensão dos voos internacionais. O esvaziamento dos aeroportos foi um dos impactos visíveis a partir da adoção dos protocolos de segurança orientados pela Organização Mundial de Saúde (OMS) e pela Organização Internacional de Aviação Civil (ICAO), que "elaboraram recomendações para orientar o funcionamento do transporte aéreo no contexto da pandemia, incluindo fechamento de fronteiras aéreas e diminuição do fluxo de passageiros atendendo recomendações de distanciamento dentro das aeronaves" (Oliveira e Castilho, 2020, p. 5).

\section{CONSIDERAÇÕES FINAIS}

A crise sanitária e econômica gerada pela pandemia da COVID-19, em nível global, afetou os empregos, a produção e o poder de compra dos cidadãos. De acordo com o painel de casos de coronavírus do Ministério da Saúde, até o dia 11 de março de 2021, 11.277.717 casos foram registrados no Brasil, com 272.889 óbitos registados (taxa de mortalidade ${ }^{4}$ de $129,9 \%$ ) e no estado do Amazonas foram 328.763 casos registrados com 11.431 óbitos (taxa de mortalidade de $275,8 \%$ ).

No caso das atividades ligadas ao turismo, a pandemia provocou impactos das mais diversas ordens nos setores de transporte, hotelaria, alimentação e no mercado de viagens e entretenimento.

\footnotetext{
${ }^{4}$ Taxa de mortalidade $=$ mortalidade $/ 100$ mil habitantes.
} 
No estado do Amazonas, diversos eventos foram cancelados, como, por exemplo, o Festival Folclórico de Parintins, o Festival de Cirandas de Manacapuru, o Festival da Canção de Itacoatiara e o Festival Amazonas de Ópera. O cancelamento destes eventos gerou reflexos em todo o setor de turismo do estado além de impactar diretamente na economia dos municípios onde ocorreriam os eventos.

Ainda que a Lei da Transparência estabeleça que os municípios devem disponibilizar as informações sobre a execução orçamentária e financeira, não foi possível consultar o todos os valores e o detalhamento sobre as finanças públicas de alguns municípios. A indisponibilidade de dados tornou a análise deficiente. No entanto, alguns investimentos em infraestrutura e obras nos municípios não pararam e espera-se que proporcionem efeitos positivos em curto e médio prazos.

As dificuldades encontradas na pesquisa foram o levantamento de dados precisos sobre os municípios, em face da ausência de uma padronização na divulgação daqueles disponíveis no portal da transparência, ou mesmo a ausência completa de informações. Outra dificuldade foi a precariedade da internet nos municípios do interior do estado, o que impediu a realização das entrevistas de modo virtual.

Com relação ao aumento no número de empresas do setor, depreende-se que a regularização das empresas poderia ter ocorrido em função do oferecimento de benefícios para o setor, em nível governamental.

No setor de empregos, foram levantados os dados sobre emprego formal nas ACT. No período estudados, apenas dois municípios tiveram mais contratações em 2020 do que em 2019: Iranduba com sete novos postos e Rio Preto da Eva com um novo posto de trabalho. Barcelos não apresentou nenhuma admissão no período analisado. No caso dos desligamentos, seis municípios apresentaram número menor de desligamentos em 2020 comparado com 2019, entre eles Barcelos que não teve nenhum desligamento, Itacoatiara, Manacapuru, Parintins, Presidente Figueiredo e Rio Preto da Eva. No entanto, a informalidade no turismo é uma realidade e os números mostram apenas parcialmente os impactos da pandemia em cada um dos municípios estudados.

E, por fim, ao analisar os dados de fluxo aéreo no estado, observa-se que o número de voos diminuiu e consequentemente houve redução no número de pessoas que embarcaram ou desembarcaram. As maiores reduções no número de voos (chegadas e partidas) ocorreram em Parintins, Tabatinga e Tefé e as maiores reduções de embarques e desembarques entre ocorreram em Parintins, Manaus (relativo a voos internacionais) e Tabatinga.

Espera-se que, no pós-pandemia, o turismo doméstico e de curta distância possa contribuir para aumentar o número de empresas e empregos nas atividades relacionadas ao setor turístico nos municípios do interior do estado. Na opinião de alguns autores (PANOSSO NETTO et al, 2020; BRITO-HENRIQUES, 2020), o 
fluxo turístico doméstico deverá ser o primeiro a ser retomado, as pessoas deverão fazer viagens mais curtas, um turismo de proximidade, valorizando, portanto, os roteiros mais locais e regionais com menos dependência do transporte aéreo.

Além disso, a intervenção governamental, por meio do desenvolvimento de uma rede de segurança social, deve ser uma prioridade para fazer face aos desafios do turismo (Higgins-Desbiolles, 2020).

Embora o isolamento social tenha se mostrado importante para reduzir a difusão da doença, outras medidas devem ser reforçadas como as ações governamentais conjuntas, integradas, nos três níveis de gestão, municipal, estadual e federal. No estado do Amazonas, estas ações deverão estar diretamente ligadas à aplicação de recursos financeiros direcionados à população mais vulnerável do estado, ampliação dos recursos humanos e físicos nos serviços públicos de saúde, melhorias no transporte com a ampliação da agilidade no atendimento dos pacientes do interior (ALEIXO et al, 2020).

Considerando que os municípios analisados apresentam estruturas sanitárias insuficientes para identificação de casos de forma rápida, para o controle e tratamento de COVID-19, os protocolos de biossegurança com medidas para a retomada da atividade turística apresentam-se comprometidos, uma vez que somente Manaus possui toda a rede de Unidades de Terapia Intensiva (UTI) no Estado do Amazonas.

De modo geral, tanto os roteiros culturais e naturais convencionais, como aqueles operados por comunidades tradicionais e povos indígenas como a pesca esportiva, a visitação às áreas protegidas, aos rios, florestas, observação de fauna e flora, foram paralisados ao longo da pandemia. Em alguma medida as perdas e os impactos foram devastadores para o turismo regional. A falta de políticas claras de apoio ao turismo, aos empreendedores, às empresas do setor ou para a retomada da atividade, coloca em risco as conquistas realizadas por estes setores, ainda que existam planos de contingência e protocolos de visitação elaborados coletivamente.

Uma nova maneira de desenvolver as atividades turísticas deve surgir, provavelmente irá ocorrer de forma local e regional, valorizando a questão de proximidade. No entanto, a recuperação da confiança do viajante exigirá muito de todas as atividades do setor do turismo nesse novo cenário

O turismo, sem nunca ter se configurado como prioritário nos investimentos e nas políticas do estado, mostra-se como uma atividade incipiente em grande parte dos municípios, que possuem desafios frente à escassez de recursos, às dimensões territoriais da região, à infraestrutura, à logística e à ausência de produtos turísticos e serviços consolidados no mercado.

Diante do exposto, seguem algumas propostas para o turismo do estado: possibilidade de gestão integrada entre órgãos da saúde e do turismo, em nível municipal, estadual e federal, com o intuito de discutir 
a retomada segura das atividades; promoção de campanhas de sensibilização para o turismo, para o turista; ampliação do acesso e da capacidade de atendimento à saúde nos municípios; ofertas contínuas de apoio às iniciativas locais e aos roteiros não convencionais de turismo; fortalecimento dos municípios com a criação de secretarias de turismo, de conselhos municipais e empenho para a criação do Fundo Municipal de Turismo e, a integração das iniciativas pública e privada na gestão municipal.

\section{AGRADECIMENTOS}

Este estudo é fruto do grupo de pesquisas "Turismo em Tempos de Pandemia: uma abordagem geográfica multi e trans-escalar". Agradecemos todo trabalho, empenho e colaboração dos pesquisadores brasileiros e estrangeiros que se uniram com o objetivo estudar os impactos da pandemia do novo coronavírus no setor do turismo. Em especial agradecemos à equipe que desenvolveu os procedimentos metodológicos (Admilson Alcântara da Silva, Carolina Todesco, Daniel de Renzo Barretti, Hugo Rogério Hage Serra, José Júlio Júnior Guambe, Maria Goretti da Costa Tavares, Rita de Cássia Ariza da Cruz, Sara Pugliesi Larrabure e Simone Affonso da Silva ) e aos demais membros da equipe que se dedica aos estudos do estado do Amazonas (Cristiane Barroncas Maciel Costa Novo, Glaubécia Teixeira da Silva, Josiani Nascimento da Silva, Katiuscia da Silva Auzier Antunes, Mayra Laborda Santos).

\section{REFERÊNCIAS}

ALEIXO, N. C. R., SILVA NETO, J. C. A. DA; PEREIRA, H. S., BARBOSA, D. E. S., LORENZI, B. C. Pelos caminhos das águas: a mobilidade e evolução da COVID-19 no estado do Amazonas. Confins. Revue franco-brésilienne de géographie/Revista franco-brasilera de geografia, n. 45, 2020. Disponível em: <https://journals.openedition.org/confins/30072\#quotation>. Acesso em 10 mar. 2021.

ALEIXO, N. C. R.; SILVA NETO, J. C. A. DA. A cartografia dinâmica da COVID-19 no Amazonas. Atlas ODS Amazonas. 18 de maio de 2020. Disponível em: <https://www.atlasodsamazonas.ufam.edu.br/ultimas-noticias/114-a-cartografia-dinamica-da-covid-19-noamazonas.html>. Acesso em: 06 mar. 2021.

ALEIXO, N. C. R.; SILVA NETO, J. C. A. DA; CATÃO, R. DE C. A difusão temporo-espacial da COVID-19 no Amazonas. Hygeia - Revista Brasileira de Geografia Médica e da Saúde, p. 336 - 348, 23 jun. 2020. Disponível em: <http://www.seer.ufu.br/index.php/hygeia/article/view/54536>. Acesso em: 06 mar. 2021.

ALLIS, Thiago; DE PAULA, Angela Teberga. Confinamento de trabalhadores de cruzeiros marítimos em tempos de pandemia: uma análise à luz das mobilidades. Revista Acadêmica Observatório de Inovação do Turismo, v. 14, n. 4, p. 50-72, 2020. Disponível em: <http://publicacoes.unigranrio.edu.br/index.php/raoit/article/view/6660 >. Acesso em: 23 mar. 2021.

ALMEIDA, C.; LÜCHMANN, L,; MARTELLI, C. A pandemia e seus impactos no Brasil. Middle Atlantic Review of Latin American Studies, v. 4, n. 1, p. 20-25, 2020. Disponível em: <https://www.researchgate.net/profile/Carla-Almeida-

17/publication/342653340_A_pandemia_e_seus_impactos_no_Brasil/links/5f443492299bf13404eef669/A-pandemia-e-seusimpactos-no-Brasil.pdf>. Acesso em: 06 mar. 2021.

BABA, C.A., STĂNCIOIU, A.-F., GABOR, M. R., ALEXE, F.A., OLTEAN, F. D., \& DINU, A. C. Considerations regarding the effects of COVID19 on the tourism market. Theoretical and Applied Economics, v. 27, n. 3, p. 624, 2020. Disponível em: < http://store.ectap.ro/articole/1489.pdf >. Acesso em 06 mar. 2021. 
BARÉ, M., BARRA, C., SOBREIRO, T., FONTOURA, A. G., ESTUPINAN, G., RADLER, J. Os riscos do turismo no rio Negro em meio à pandemia. Disponível em: <https://www.socioambiental.org/pt-br/blog/blog-do-rio-negro/os-riscos-do-turismo-no-rio-negro-emmeio-a-pandemia>. Acesso em 10 mar. 2021.

BLANCO, Jorge. Territorio, circulación y redes: articulaciones y tensiones. In: Arroyo, M. e Cruz, R. C. A (org.). Território e circulação: A dinâmica contraditória da globalização. São Paulo: FAPESP/PPGH/CAPES/Annablume, 2005.

BRASIL. Lei Complementar no 131, de 27 de maio de 2009. Disponível em: <http://www.planalto.gov.br/ccivil_03/leis/lcp/lcp131.htm>. Acesso em 10 mar. 2021.

BRITO-HENRIQUES, Eduardo. COVID-19, turismo e sustentabilidade: tudo está interligado. Finisterra, v. 115, n. 55, 2020. Disponível em: <https://revistas.rcaap.pt/finisterra/article/view/20311>. Acesso em: 06 mar. 2021.

CRUZ, Rita de Cássia Ariza da. O evento da COVID-19 e seus impactos sobre o setor turismo: em busca de uma análise multi e transescalar. Revista Acadêmica Observatório de Inovação do Turismo, [S.I.], p. 1-15, dez. 2020. ISSN 1980-6965. Disponível em: <http://publicacoes.unigranrio.edu.br/index.php/raoit/article/view/6636/3343>. Acesso em: 06 mar. 2021.

FGV - Fundação Getúlio Vargas. Impacto econômico do Covid-19 - Propostas para o turismo. Rio de Janeiro: FGV, jun. 2020. Disponível em:

<https://fgvprojetos.fgv.br/sites/fgvprojetos.fgv.br/files/02.covid19_impactoeconomico_turismo2_v07_fichacatalografica.pdf>. Acesso em 06 mar. 2021

FVS/AM - Fundação de Vigilância em Saúde do Amazonas. Coronavírus: Perfil clínico e demográfico dos casos de COVID-19 no estado do Amazonas: uma análise comparativa entre 2020 e 2021. ANO 2, Boletim № 17, 2021. Disponível em: <http://www.fvs.am.gov.br/media/publicacao/boletim_covid_17.pdf>. Acesso em: 06 mar. 2021.

GÖSSLING, Stefan; SCOTT, Daniel; HALL, C. Michael. Pandemics, tourism and global change: a rapid assessment of COVID-19. Journal of Sustainable Tourism, v. 29, n. 1, p. 1-20, 2020. Disponível em:

<https://www.tandfonline.com/doi/full/10.1080/09669582.2020.1758708>. Acesso em: 06 mar. 2021.

HIGGINS-DESBIOLLES, Freya. Socialising tourism for social and ecological justice after COVID-19. Tourism Geographies, v. 22, n. 3, p. 610-623, 2020. Disponível em: <https://www.tandfonline.com/doi/full/10.1080/14616688.2020.1757748>. Acesso em 06 mar. 2021.

HARVEY, David. Anticapitalismo em tempos de pandemia: marxismo e ação coletiva. Boitempo Editorial, 2020.

IBGE. Cidades. Manaus Disponível em: <https://cidades.ibge.gov.br/brasil/am/manaus/panorama>. Acesso em: 06 mar. 2021.

IBGE. Geociências - Downloads. 2021. Disponível em: < https://www.ibge.gov.br/geociencias/downloads-geociencias.html>. Acesso em 06 mar. 2021.

IPEA - Instituto de Pesquisa Econômica Aplicada. Mercado de Trabalho conjuntura e análise. Brasília (DF): IPEA, julho, 2020 Disponível em: <https://www.ipea.gov.br/portal/images/stories/PDFs/mercadodetrabalho/200811_bmt\%2069_web.PDF> Acesso em 06 mar. 2021.

OLIVEIRA, Bruno Carneiro de; CASTILHO, Denis. Entre fluxos e escalas: COVID-19 e suas implicações na rede de transporte aéreo no primeiro semestre de 2020. GEOgraphia, v. 22, n. 49, 2020. Disponível em:

<https://periodicos.uff.br/geographia/article/view/45882>. Acesso em 10 mar. 2021.

PANOSSO NETTO, A., OLIVEIRA, J. L. S., SEVERENI, V. Do overtourism à estagnação. Reflexões sobre a pandemia do Coronavírus e o turismo. Cenário: Revista Interdisciplinar Em Turismo e Território, 8 (14), 26-43. 2020. Disponível em:

<https://periodicos.unb.br/index.php/revistacenario/article/view/32002/26352>. Acesso em: 06 mar. 2021.

SCHOR, Tatiana, COSTA, Danielle Pereira da. Rede urbana na Amazônia dos grandes rios: uma tipologia para as cidades na calha do rio Solimões-Amazonas - AM. Anais do X SIMPURB, Florianópolis, 2007. Disponível em:

$<$ http://www.dpi.inpe.br/Miguel/UrbisAmazonia/Dialogos_Norte-

Nordeste/Texto_TatianaSchor_RedeUrbAmz_CalhaSolimoes_2006.pdf>. Acesso em: 06 mar. 2021.

SCHOR, Tatiana; DE OLIVEIRA, José Aldemir. Reflexões metodológicas sobre o estudo da rede urbana no Amazonas e perspectivas para a análise das cidades na Amazônia Brasileira. Acta Geográfica, v. 5, n. 11, p. 15-30, 2011. Disponível em:

<https://revista.ufrr.br/actageo/article/view/539 >. Acesso em: 23 mar. 2021.

SEBRAE. Serviço Brasileiro de Apoio às Micro e Pequenas Empresas. Análise da crise e impactos para os pequenos negócios. SEBRAE/ES. 1ạd. 2020. Disponível em:

<https://www.sebrae.com.br/Sebrae/Portal\%20Sebrae/UFs/ES/Sebrae\%20de\%20A\%20a\%20Z/RELAT\%C3\%93RIO\%20IMPACTO\%20C OVID\%20-\%20CORRETO.pdf>. Acesso em 06 mar. 2021. 
SEDECTI - SECRETARIA DE ESTADO DE DESENVOLVIMENTO ECONÔMICO, CIÊNCIA, TECNOLOGIA E INOVAÇÃO. Perfil do Emprego Formal no Setor do Turismo no Amazonas. 2018. Disponível em: <http://www.sedecti.am.gov.br/indicadores-mapa/>. Acesso em 10 mar 2021.

SPÓSITO, Maria Encarnação Beltrão. Novas redes urbanas: cidades médias e pequenas no processo de globalização. Geografia, v. 35 , n. 1, p. 51-62, 2010. Disponível em: <https://www.periodicos.rc.biblioteca.unesp.br/index.php/ageteo/article/view/4817>. Acesso em 23 mar. 20121.

TRIGO, Luiz Gonzaga Godoi. Viagens e turismo: dos cenários imaginados às realidades disruptivas. Revista Brasileira de Pesquisa em Turismo, v. 14, n. 3, p. 1-13, 2020. Disponível em: < https://www.rbtur.org.br/rbtur/article/view/2107> Acesso em: 20. mar. 2021.

UNWTO - World Tourism Organization. Barometer May 2020: Special focus on the Impact of COVID-19. May 2020. Disponível em: <https://webunwto.s3.eu-west-1.amazonaws.com/s3fs-public/2020-05/Barometer May2020 full.pdf>. Acesso em 06 mar. 2021.

WHO - World Health Organization. A World at Risk. Annual report on global preparedness for health emergencies Global Preparedness Monitoring Board. September 2019. Disponível em:

<https://apps.who.int/gpmb/assets/annual report/GPMB annualreport 2019.pdf>. Acesso em 06 mar. 2021.

YANG, Yang; ZHANG, Hongru; CHEN, Xiang. Coronavirus pandemic and tourism: Dynamic stochastic general equilibrium modeling of infectious disease outbreak. Annals of Tourism Research, 2020. Disponível em:

<https://www.ncbi.nlm.nih.gov/pmc/articles/PMC7147856/>. Acesso em 06 mar. 2021.

ZORZETTO, Ricardo. Epidemiologia. Novo coronavírus começou a se espalhar no Brasil entre janeiro e fevereiro. Pesquisa Fapesp, 12 maio 2020. Disponível em: <https://revistapesquisa.fapesp.br/novo-coronavirus-comecou-a-se-espalhar-no-brasil-entre-janeiro-efevereiro/>. Acesso em: 06 mar. 2021. 